\title{
Drug targeting strategies for liver cancer and other liver diseases
}

\begin{abstract}
Treatment of liver cancer and other diseases are a challenging task for the current researchers in the pharmaceutical field. There are some physiological barriers like RES uptake, opsonization and first-pass metabolism of therapeutics present that make drug therapy more complex. Generally, conventional cancer therapy approaches give low response rate or remain un-success due to multi drug resistance (MDR), high clearance rate, severe adverse effect due to unwanted drug distribution and in-adequate concentration reached in cancer cells. Therefore it is a need to develop novel strategies which will target drug molecule specific to affected liver cells. In the current era of research and development, various approaches have been utilized to improve the drug delivery and drug targeting. The new targeting approaches are based on the use of targeting ligands which can conjugate with nanocarrier or with drug molecules. These conjugates systems are accumulate passively or actively. This review focus on some liver cancer cells specific targeting ligands such as manos6 phosphate, asialoglycoprotein, galactoside, lactobionic acid, PDGF, antibodies, aptamers, avimers which have been utilized to target cancer cell after conjugation with the therapeutic system. This review also describes the novel targeting approaches including latest targeting molecules and targeted drug delivery system used for the treatment of liver cancer.
\end{abstract}

Keywords: reticuloendothelial organ, hepatocellular, kupffer cells, hepatocytes, liposomes
Volume 2 Issue 4 - 2018

\author{
Satish Shilpi,' Roshni Shivvedi,' Ekta \\ Gurnany,' Sonal Dixit,' Kapil Khatri,' Deepak \\ K Dwivedi \\ 'Department of Pharmaceutics, Ravishankar College of \\ Pharmacy, India \\ ${ }^{2}$ Department of Pharmaceutics, Dr. H.S. Gour University, India
}

Correspondence: Satish Shilpi, Department of Pharmaceutics, Ravishankar College of Pharmacy, India, Tel +91940652069l, Email shilpisatish@gmail.com

Received: June 12, 2018| Published: July 03, 2018

\section{Introduction}

Liver diseases, particularly hepatitis-B (viral HBV infections), liver fibrosis and hepatocellular carcinoma ( $\mathrm{HCC})$ are the major causes of disability and mortality worldwide. ${ }^{1}$ These require long-term drug therapy. Liver-specific drug delivery is helpful in decreasing side effects by reducing drug distribution in non-target organ and improves the therapeutic efficacy by concomitantly increasing the drug concentration in target cells. ${ }^{2}$ As the liver is the largest reticuloendothelial organ in the body, macrophages in the liver (i.e. kupffer cells) are attractive candidates to serve as the effector cells for therapy of hepatic diseases. ${ }^{3,4}$ The body distribution and opsonisation of colloidal drug carrier systems by macrophages seem to be influenced by their particle sizes and surface characteristics. ${ }^{5}$ The feasibility of particles ranged in 50-200nm to arrive at fenestration in the hepatic sinusoidal endothelium might lead to hepatic accumulation after IV injection. Although the carrier systems like nano particles or liposomes could not directly reach the hepatocytes, the uptake of IV injected particulate drug carriers by macrophages cells might as well be the main limiting factor in the efficient targeting of a drug to the kupffer cells in the liver. ${ }^{6}$

\section{Basic epidemiology of liver diseases}

\section{Hepatitis-B}

Hepatitis-B is caused by a DNA virus; it is transmitted through parenteral or mucosal exposure to infected blood, serous fluids and other body fluids such as seminal and vaginal fluids. Perinatal transmission (from an infected mother to infant during birth), unsafe needle sharing, blood transfusion practices and sexual contact are common routes of infection. Hepatitis-B is of two types acute and chronic. Chronic HBV infection can be divided in to three major phases based on virus host interactions, immune tolerant, immune clearance and inactive carrier phase. ${ }^{7}$ Characteristic symptoms of immune tolerant phase is seropositivity of hepatitis-B antigen ( $\mathrm{HBe}$ $\mathrm{Ag}$ ), active viral replication (elevated HBV DNA blood levels) with little or no evidence of liver inflammation [i.e. Normal serum alanine aminotransferase (ALT) levels. In the immune clearance phase, liver inflammation occurs (elevated serum ALT levels). In this phase, antiviral drugs are used to reduce the risk of developing liver cirrhosis and HCC. Clearance of $\mathrm{HBeAg}$ and the development of anti-HBeAg antibodies (HBeAg seroconversion), occurs in inactive carrier phase which is accompanied by a concomitant reduction in HBV DNA levels and normalization of serum ALT levels with improvement in liver fibrosis and inflammation over time. ${ }^{8}$ Chronic hepatitis B also can lead to a type of liver cancer known as hepatocellular carcinoma.

\section{Liver fibrosis}

Chronic liver injury and persistent wound healing occur in liver fibrosis that could lead to cirrhosis i.e fibrosis is a wound healing process of liver in the body in which extracellular matrix (ECM) proteins and collagen fiber are formed that deposits and retain in liver tissue and responsible for causing tissue scar. Some other complications are also encountered during tissue injury such as loss of hepatic functions, ascites, portal hypertension, increased risk for esophageal varices and HCC. HCC is a most serious complication that often fatal. Drug or toxin-induced injury, chronic alcoholism, viral hepatitis, prolonged biliary obstruction and inherited metabolic disorders such as Willson's disease and hematochromatosis are main causes of liver fibrosis. ${ }^{8}$ Cellular and molecular mechanisms of liver fibrosis has greatly advanced. Activated hepatic stellate cells (HSCs), 
portal fibroblasts, and myofibroblasts of bone marrow origin have been identified as major collagen-producing cells in the injured liver. These cells are activated by fibrogenic cytokines such as TGF- $\beta 1$, angiotensin II, and leptin. Cytokines such as transforming growth factor- $\beta$ (TGF- $\beta$ ) and platelet-derived growth factor (PDGF) contribute to HSC activation and proliferation responsible for activation of myofibroblasts. Some other cytokines, transcription factors and intracellular signaling are also involve in this process. Liver fibrosis can be controlled by inhibiting the activation of HSCs.

\section{Hepatocellular carcinoma}

Liver diseases such as chronic hepatitis-B or $\mathrm{C}$ virus infections, alcoholic cirrhosis and non-alcoholic steatohepatitis, if occurs for a long time, results in HCC. ${ }^{9}$ The development of HCC from each of the chronic liver diseases is a complex and multistep process with an accumulation of genetic and epigenetic alterations, which culminates in the aberrant activation of molecular signalling pathway connected to cellular proliferation and survival, as the central themes. ${ }^{10}$ Hepatitis-C is a single-stranded RNA virus, having high genetic variability. There are six different genotypes of HCV isolated i.e genotype-I, II, III, IV, $\mathrm{V}$ and VI. Simultaneously infection of Hepatitis-B and Hepatitis-C virus in a patient liver who already suffered from cirrhotic has increases the chances of HCC. ${ }^{11-18}$

\section{Treatments of various liver diseases}

\section{Hepatitis-B therapy}

$\mathrm{HBeAg}$ seroconversion and by reduction of the circulating HBV DNA levels results in retardation of active HBV replication, which is a helpful tool to treat liver injuries and liver disease progression. The seven U.S. Food and Drug Administration (FDA) approved anti-HBV drugs can be broadly categorized as interferons (IFN) (IFN- $\alpha 2 b$ and pegylated IFNa2a), nucleoside (lamivudine, entecavir and telbivudine) and nucleotide (adefovir and tenofovir) analogs. Proposed the use of monostearin containing solid lipid nanoparticles (SLN) as an effective drug delivery system for Adefovir and Dipivoxil in HepG2.2.15 cells, leading to significant down regulation of DNA levels in HBsAg, $\mathrm{HBeAg}$ and $\mathrm{HBV}$ in comparison to the free drug which leads to an enhanced reduction in the viral loads in the liver. Direct drug delivery to liver shown less systemic side effects. Reported that incorporation of a derivative of the nucleoside analogue iododeoxy-uridine into recombinant chylomicrons leads to selective targeting to liver parenchymal cells that was achieved effective and higher intracellular drug concentrations in liver. These drug carrier complexes represent a conceptual advance in the development of an effective and safe therapy for hepatitis-B. demonstrated a hepatocyte targeted system i.e. N-acetylgalactosamine-conjugated melittin-like peptide (NAGMLP) with potent cholesterol-conjugated siRNA (a targeting coagulation factor VII) that are capable to target conserved HBV sequences resulted in multilog repression of viral RNA, proteins, and viral DNA. These conjugates holds great promise as a new therapeutic for patients chronically infected with HBV.

\section{Liver fibrosis therapy}

No standard treatment is available for liver fibrosis due to the complex and varied etiologies of liver fibrosis. Anti-fibrotic therapy differs depending on the type of liver disease. Combination of pegylated IFN and ribavirin used in the treatment of liver fibrosis. ${ }^{11}$ Alcohol asceticism is an efficient way to recover alcohol- induced liver fibrosis. ${ }^{12}$ For treatment of autoimmune hepatitis and acute alcoholic hepatitis, corticosteroids are used because of inflammatory nature of the disease. ${ }^{13}$ Rennin-angiotensin system inhibitors, IFN- $\gamma$, peroxisomal proliferator-activated receptor (PPAR)- $\gamma$ ligands, pirfenidone, colchicines and other herbal medicines are also used for the treatment of liver fibrosis. ${ }^{13,14}$ Reported that combined delivery of sorafenib and a MEK inhibitor via CXCR4-targeted nano particle to prevent activation of ERK in activated hepatic satellite cells (HSCs). It was shown anti-fibrotic effects in the CCl4-induced murine model. Targetable HSCs represents a promising strategy to prevent the development and progression of fibrosis-associated HCC. Demonstrated that silibinin and siColl $\alpha 1$ loaded Vitamin Adecorated biocompatible micelles which was safe and efficient HSCstargetable chemogene-delivery system for inhibiting fibrous collagen I and may serve as a novel and effective clinical option for treatment of liver fibrosis. Prepared polypeptide pPB-modified stable nucleic acid lipid nanoparticles (pPB-SNALPs) to selectively deliver siRNAs against heat shock protein 47 to the liver for hepatic fibrosis therapy. The study was shown increased uptake of prepared system by LX-2 cells and primary hepatic stellate cells (HSCs) of mice. It was shown increased liver distribution and HSC uptake in vivo. In addition, pPBSNALP also exhibited an enhanced inhibitory effect on TAA-induced hepatic fibrosis in mice model with high gp46 mRNA expression.

\section{Anti-HCC therapy}

Surgical resection and liver transplantation is only treatment of HCC in the early stage of the disease. Nonsurgical local ablation techniques such as percutaneous ethanol injection and radiofrequency ablation are also used in HCC treatment. ${ }^{15}$ The single liver tumor can be removed by surgical resection but an inadequate supply of donor organ is a limiting factor for it. In the case where surgical resection or transplantation is not suitable, nonsurgical local ablation treatments are very useful. Percutaneous ethanol injection is an intra-tumoral injection of absolute ethanol is given with use of ultrasound. It causes tumor mass dehydration and necrosis. RFA is the percutaneous delivery of radiofrequency energy via single or multiple electrodes which results in thermal necrosis of the cancerous tumor. ${ }^{16}$ Some drugs also used in the treatment of HCC like doxorubicin, cisplatin and 5fluorouracil, used alone or in combination. Grafted Xyloglucan with the doxorubicin (DOX) and galactosamine to target liver hepatocytes ${ }^{1}$. In vitro cytotoxicity experiment revealed that similar cytotoxicity as free DOX against HepG2 cells and when it incubated with HeLa cells there was no significant cytotoxicity change while in case of a human tumor xenograft nude mouse model, the prepared conjugates shown higher therapeutic effect than without conjugated and plain doxorubicin. They revealed that the prepared conjugates was improved the transfection efficiency and hepatocyte specificity that can be for tumor therapy. Demonstrated mechanism of GG-8-6 (1) induced apoptosis, G2/M arrest of, and the activation of caspase pathways in HCC cells. In vivo anti-tumor experiments showed that GG-8-6 (1) could significantly inhibit the growth of tumor in the mouse xenograft tumor model. At the dose of $40 \mathrm{mg} / \mathrm{kg}$ of GG-8-6 (1), the inhibition ratio was $67.9 \%$ without weight loss. These finding suggested that GG-8-6 (1), a new cyclic peptide, might be a potential candidate for developing new anti-HCC drug in the coming future. Prepared antibody conjugated nano particle containing Sorafenib which was shows higher cellular uptake by HepG2 cells than without antibodyconjugated nanoparticles. The prepared system was down regulated the expression of anti-apoptosis molecule MCL-1, which resultant in polymerization of Bax. It was promoted the mitochondrial release of cytochrome C, resulting in cellular apoptosis. Moreover, 
the antibodies conjugated nanoparticles significantly inhibited the growth of HepG2 xenograft tumors in nude mice without producing evident side effects. Developed calcium phosphate nanoparticles were loaded with FTY720 and siRNA. These NPs were stable in systemic circulation and easily up taken by affected cells due to their nano metric size. Furthermore, co-delivery of FTY 720 and Beclin 1 siRNA significantly increased cytotoxicity in vitro and in vivo compared with that caused by treatment with the free drug alone.

\section{Liver-specific targeting}

Different carrier systems like liposomes, nano particles when entering into the bloodstream, nonspecific interaction occur with serum proteins and surface deposition of antibodies/complement proteins, this process called opsonization. This interaction results reduction of overall dose and reduction of circulation time of carriers via mechanical entrapment of aggregates in the alveoli and clearance by reticuloendothelial system in liver, spleen and bone marrow especially if the size is greater than $200 \mathrm{~nm}$ and a large surface negative charge is present. The endothelial cells lining the liver sinusoids are another component of the RES h scavenger receptors that can internalize particles up to $0.23 \mu \mathrm{m}$ in vivo. Steric stabilization and shielding of carriers by incorporating PEG moieties minimizes protein binding and reduce non-specific scavenging of carriers by RES. ${ }^{17}$ Generally targeting achieved by passive and active targeting. Passive targeting is obtained through desirable size and surface modified nanocarriers. Site-specific delivery of therapeutics can be obtained by passive targeting which increases the local concentration of the drug and reduces undesirable side effects. Active targeting is obtained by surface modification of nano particles with specific ligands such as carbohydrates, peptides, proteins and antibodies, which has the benefit of facilitating uptake in a liver cell type-specific manner, hence minimizing alterations to the physiological functions of other liver cell types.

\section{Passive targeting}

Nano particle therapeutics accumulates at specific body site due to certain anatomic or patho physiological features, this type of targeting known as passive targeting. ${ }^{18}$ Liver sinusoids capillaries have two special characteristics first $100-200 \mathrm{~nm}$ fenestrations present along the endothelial wall and second is the absence of basal lamina. These characteristics facilitate the passive accumulation of nano particle therapeutics. Nano particle therapeutics having a diameter less than $200 \mathrm{~nm}$ facilitates passive liver targeting because these particles can extravasate through the slightly larger sinusoidal fenestrations, due to this process nano particle therapeutics obtained high local concentration at space of disuse, where distribution to the various liver cell types can occur. ${ }^{19}$ EPR effect that was first described by Matsumura and Maeda in 1986, also helps in passive accumulation of nano particle therapeutics in the liver. Tumour has some distinctive features which increase EPR effect; these are leaky tumour vasculature leading to rapid and incomplete tumour angiogenesis to meet the elevated demands for oxygen and nutrients that results in enhanced permeability and extravasation of macromolecules, and impaired lymphatic drainage, which favors the retention of nano particle therapeutics in the tumour tissues. ${ }^{20}$ Endothelial cells have gap junction size of between 400 and $600 \mathrm{~nm}$, therefore nano particle therapeutics expected to be extremely efficient at extravagating from the tumour microvasculature to result in a high local tumour interstitial concentration. ${ }^{21,22}$

\section{Active targeting}

Site-specific delivery of therapeutic system increases the local concentration of the drug, due to which therapeutic efficiency of drug increases and unwanted side effects decreases. Non-parenchymal sinusoidal endothelial cells (SECs), kupffer cells (KCs), hepatic stellate cells (HSCs) and the predominant parenchymal hepatocytes are responsible for diverse physiological functions of the human liver. HSCs are the main target in liver fibrosis because they are responsible for secretion and maintenance of copious amounts of extracellular matrix (ECM) in response to various biochemical stimuli produced by the injured hepatocytes, SECs and KCs. Hepatocytes are the main target for $\mathrm{HBV}$ infections and $\mathrm{HCC} .{ }^{23}$ Targeted drug delivery systems is very useful treatment of many liver diseases such as chronic viral hepatitis, liver fibrosis and hepatocellular carcinoma and it also beneficial to reduce side effects by reducing drug distribution in non-target cells and improve the therapeutic efficacy by concomitantly increasing the drug concentration in target cells. Sugar moieties are commonly used to target asialoglycoprotien receptors in hepatocytes mannose receptors in kupffer and liver endothelial cells. Some ligand-mediated approaches for liver cell targeting

1. Mannose 6-phosphate receptors present on hepatic stellate cells, by using mannose 6 -phosphate as ligand we can target the liver, this strategy used in the treatment of liver fibrosis. ${ }^{24-25}$.

2. Retinol binding protein receptors present in hepatic stellate cells, vitamin-A used as a ligand to treat liver fibrosis. 26

3. Type VI collagen receptor and PDGF receptor present on hepatic satellite cells are highly expressed in liver fibrosis, Cyclic RGD and PDGF respectively used as a ligand in the treatment of liver fibrosis. ${ }^{27}$

4. Scavenger receptor class A is targeted by human serum albumin present on hepatic stellate cells also useful in liver fibrosis treatment.

5.Asialoglycoprotien receptors present on hepatocytes. Asialoorosomucoid, Galactoside,28,29 Galactosamine.30 Asialofetuin,31 Sterylglucoside,32 Lactose/lactobionic acid,33,34 PVLA.35 used as a ligand to target these receptors, this strategy used in the treatment of HCC.36

6. Scavenger receptor class B type I receptors present on hepatocytes, which are highly expressed in hepatoma cells and tumors, Apolipoprotein A- I used as a ligand to target these receptors. ${ }^{37}$

7. Plasma membrane fatty acid binding protein (Putative) are targeted by Linoleic acid, these receptors also present on hepatocytes and used in the treatment of $\mathrm{HC} .{ }^{38}$

8.Glycyrrhizin receptors are targeted by Glycyrrhizin and Heparan sulphate by Acetyl-Cknekknkiernnklkqpp-amide ligand; both receptors present on hepatocytes and used to treat HCC. ${ }^{39}$

\section{Other approaches for targeting liver cancer}

\section{Targeting carboxyl esterases (CES) over expressed by cancerous liver cells}

Since in liver cancer cells carboxyl esterase-1 (CES-1) \& carboxyl esterase-2 (CES-2) are over expressed, the derivative of the drugs converted to active form by CES -1 or CES -2 in the liver, thus 
an active form of the drug is available to target cancer cells of the liver. Carboxyl esterases are serine esterases that cause hydrolysis of various esters and carbamates. Therefore carbamate prodrugs of some anticancer drugs are used to target liver cancer. ${ }^{40}$ Some examples of prodrugs are Carbamate prodrugs of doxazolidine, 5-FU, and camptothecin Figure 1.

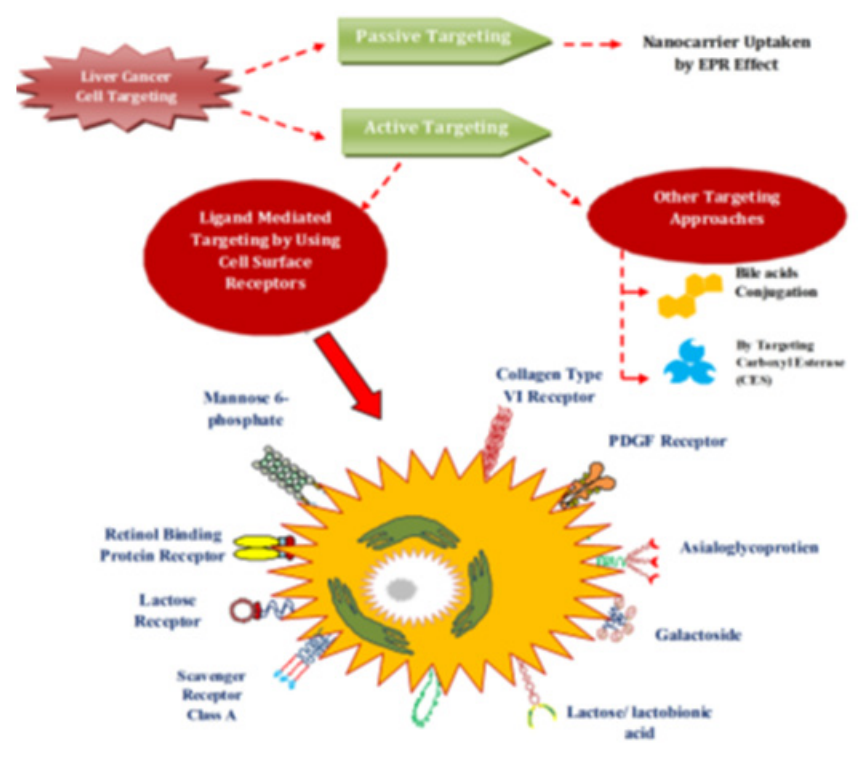

Figure I Targeting approaches for liver cancer cell.

\section{Tethering bile acids}

Another approach for targeting liver cancer is the use of bile acids, which are efficiently taken up by hepatoma cells via sodiumindependent transport carriers. A series of bile acid-platinum conjugates have been synthesized and their cytotoxicity investigated both in vivo and in vitro. Conjugate Bamet-UD2 exhibited enhanced uptake in hepatocytes and has got ability similar to that of cisplatin to inhibit tumor growth and tendency to prolong survival time. ${ }^{41}$

\section{Treating liver cancer with glass beads}

Treating liver cancer with tiny radioactive glass beads is a new method which has been recently introduced. In this treatment, radioactive glass beads injected into the main artery which supplying blood to the liver. The blood carries the beads into the liver, where they deliver localized radiation to malignant cells in liver tumors. ${ }^{42}$

\section{High density lipoprotein particles based targeting}

Use of targeting moiety increases the therapeutic effect of the drug and decreases side effects of drug and decreases side effects of the drug. ${ }^{43-45}$ Various targeting carriers are used in targeting to organs in which lipoproteins are potential drug carrier. Lipoproteins are spherical particles which are consisted of a polar lipid core surrounding phaspholipid monolayer, cholesterol and apoprotiens are embedded in it. Because core consists of a polar lipid highly hydrophobic drugs can be easily incorporated in to it. ${ }^{46}$ Lipoprotiens are endogeneous in origin so these particles are completely biodegradable and nonimmunogenic and also RES system is unable to recognize them. HDL is an important class of lipoproteins which is transport cholesterol from various tissues to the liver. There is specific recognition receptor SR-BI of apolipoprotien A-I (apo A-I) on hepatocyte surface. HDL takes up cholesterol from peripheral tissues and delivers it back to the liver via apoA-I, which binds to its receptor on the liver cell membrane, ${ }^{47}$ due to this reason, HDL-drug complex is a very useful carrier for delivering antitumor drugs to hepatocyte cells.

\section{Liver targeting micelles}

Polymeric micelles have some remarkable advantages, such as small size and narrow size distribution, ${ }^{48}$ long term survival in blood circulation and sulubilization of hydrophobic drugs. ${ }^{49,50}$ Due to these advantages polymeric micelles are very useful to deliver the anticancer drug. These particles have the nano metric range and formed from self-assembled amphiphilic block co-polymers having core-cell architecture in aqueous solution. Therapeutic agents remain in this hydrophobic core and avoid possible degradation during in vivo transportation and hydrophilic shell of micelle maintain a hydration barrier that can effectively stabilize the drug-loaded micelles in the blood circulation. ${ }^{51,52}$ Silibinin and siColl $\alpha 1$ loaded Vitamin A-decorated biocompatible micelles were developed to deliver therapeutic molecules safely and efficiently to HSCs to inhibiting fibrous collagen-I for treatment of liver fibrosis.

\section{Surface modified liposome}

A liposome is a novel drug delivery system consisting of lipid layer containing water phase. Both hydrophilic and lipophilic drugs can encapsulate in to it. By modification of the surface of the liposome, drugs can be targeted to desired site or organ in the body. ${ }^{53,54}$ When liposomes administered intravenously, it is cleared by RES. ${ }^{55}$ Kupffer cells in the liver are part of RES, that's why liposome is mostly accumulated in the liver specially in non-parenchyma cells. $^{56}$ ASGP-receptor present on hepatocytes, these receptors recognised by galactose moiety attached as a ligand on the surface of liposome and they incorporated in to cells by endocytosis. Galactose terminated compounds such as lactosylceramide, ${ }^{57}$ asialofetuin, or synthetic glycolipids, are used to modify liposomes. For example sterylglucoside containing liposome accumulated in the liver, especially in hepatocytes. . $^{58,59}$

\section{Chylomicron emulsion}

Natural spherical macromolecular emulsion particles called lipoproteins, these lipoproteins are involved in intercellular lipid and cholesterol transport in the circulation. ${ }^{60}$ Chylomicrons are triglyceride-rich lipoprotein emulsions, dietary lipids absorbed through the intestine membrane in to blood circulation are packed into chylomicrons. ${ }^{61,62}$ Lipase is a lipoprotein enzyme which can hydrolyze the core triglycerides of the chylomicron in blood circulation. On the surface of the chylomicron, many different apolipoprotiens are anchored by their receptors receptors these modified chylomicrons are taken up by liver parenchymal cells. When chylomicrons are reconstituted by natural lipids $(100 \mathrm{~nm})$ was preferentially taken up by liver hepatocytes. ${ }^{63}$ When a drug is incorporated in to chylomicron emulsion it can be easily targeted to the liver. Drug inside the emulsion is chemically more stable than a free drug because it is protected from enzymatic degradation. Also, the drug shows significant release when it is inside chylomicron emulsion. ${ }^{64}$ Nucleoside analogue iododeoxyuridine was successfully delivered by recombinant chylomicrons to target liver parenchymal cells for hepatitis B treatment. It was delivered encapsulated therapeutics in higher concentrations in liver. 


\section{Magnetoliposomes}

It is a novel drug delivery carrier system..$^{65,66}$ Generally used anticancer drugs exhibit severe side effects that limit their effective therapeutic use. ${ }^{67}$ Magnetoliposome is an approach to increase the beneficial/adverse effect ratio of such drugs, which is used to target diseased organs or tissues by using a magnetic field.

\section{Conclusion}

There are many limitations are present in conventional approaches for the treatment of liver cancer. In this conditions, required some new strategy to treat or manage advanced liver cancer. This review concluded that there are some new strategies available such as ligand mediated active targeting, nano carrier based RES uptake and some molecular conjugates of targeting ligands and active drugs that applicable to target effectively and killed the actively proliferating cancer cells, ${ }^{68,69}$ and the available targeting ligands such as asialoglycoprotein, lactose and mannose, liver cancer cell specific antibodies, aptamers, avimers, bile acid conjugation, Collagen Type VI Receptor, Collagen Type VI Receptor and others ligands were successfully used for targeting liver cancer and further can be used for targeting and deliver anticancer drug or drug which are used to treatment of liver diseases. ${ }^{70}$

\section{Acknowledgements}

Author want to acknowledge the Science and Engineering Research Board (SERB), Department of Science and technology (DST), New Delhi, India and Madhya Pradesh Council of science and Technology (MPCST) for awarding research project and providing financial assistance to carry out the research work on advanced breast cancer and liver cancer.

\section{Conflict of interests}

Author declares that there is no conflict of interest.

\section{References}

1. Cao Z, Li Z, Wang Y. et al. Assessment of serum Golgi protein 73 as a biomarker for the diagnosis of significant fibrosis in patients with chronic HBV infection. J Viral Hepat. 2017;24:57-65.

2. Yuan L, Wang J, Shen W. Reversible lipidization of somatostatin analogues for the liver targeting. Eur J Pharm Biopharm. 2008;70(2):615-620.

3. Grislain L, Couvreur P, Lenaerts V, et al. Pharmacokinetics and distribution of a biodegradable drug-carrier. Int J Pharm. 1983;15(3):335-345.

4. Yamashita C, Matsuo H, Akiyama K, et al. Enhancing effect of cetylmannoside on targeting of liposomes to kupffer cells in rats. Int $J$ Pharm. 1991;70(3):225-233.

5. Harashima H, Sakata K, Funato K, et al. Enhanced hepatic uptake of liposomes through complement activation depending on the size of liposomes. Pharm. 1994;11(3):402-406.

6. Klibanov AL, Maruyama K, Torchilin VP, et al. Amphipathic polyethyleneglycols effectively prolong the circulation time of liposomes. FEBS Lett. 1990;268(1):235-237.

7. Liaw YF. Natural history of chronic hepatitis B virus infection and longterm outcome under treatment. Liver Int. 2009;29(1):100-107.

8. Li L, Wang H, Ong Z, et al. Polymer-and lipid-based nano particle therapeutics for the treatment of liver diseases. Nano Today. 2010;5(4):296-312.
9. El-Serag HB, Rudolph KL. Hepatocellular carcinoma: epidemiology and molecular carcinogenesis. Gastroenterology. 2007;132(7):2557-2576.

10. Llovet JM, Bruix J. Molecular targeted therapies in hepatocellular carcinoma. Hepatology. 2008;48(4):1312-1327.

11. Poynard T, Hutchison J, Manns M, et al. Impact of pegylated interferon alfa-2b and ribavirin on liver fibrosis in patients with chronic hepatitis $\mathrm{C}$. Gastroenterology. 2002;122(5):1303-1313.

12. Bataller R, Brenner DA. Liver fibrosis. JClin Invest. 2005;115(2):209-218.

13. Sung Yun-Chieh, Liu YC, Chao PH, et al. Combined delivery of sorafenib and a MEK inhibitor using CXCR4-targeted nano particles reduces hepatic fibrosis and prevents tumor development. Theranostics. 2018;8(4):894-905.

14. Rockey DC. Current and future anti-fibrotic therapies for chronic liver disease. Clin Liver Dis. 2008;12(4):939-962.

15. Chen JT, Ru M, Sun SC, et al. Synthesis and biological evaluation of cyclopeptide GG-8-6 and its analogues as anti-hepatocellular carcinoma agents. Bioorganic Med Chem. 2018;26(3):609-622.

16. Bruix J, Sherman M. Management of hepatocellular carcinoma. Hepatology. 2005;42(5):1208-1236.

17. Dong $\mathrm{X}, \mathrm{Gu} \mathrm{R}, \mathrm{Zhu} \mathrm{X}$, et al. Evaluating prodrug characteristics of a novel anticoagulant fusion protein neorudin, a prodrug targeting release of hirudin variant 2-Lys47 at the thrombosis site, by means of in vitro pharmacokinetics. Eur J Pharm Sci. 2018;121:166-177.

18. Magalhaes Santos NS, Mosqueira VC. Nanotechnology applied to the treatment of malaria. Adv Drug Deliv Rev. 2010;62(5):560-575.

19. Xia Q, Li L, Zhao L. Silica nano particle based dual responsive nano prodrug system for liver cancer therapy. Exp Ther Med. 2017;14(3):2071-2077.

20. Matsumura Y, Maeda H. A new concept for macromolecular therapeutics in cancer chemotherapy: mechanism of tumoritropic accumulation of proteins and the antitumor agent smancs. Cancer Res. 1986;46(12):6387-6392.

21. Nissinen Tuomo, Simo Näkki, Hanne Laakso, et al. Tailored dual PEGylation of inorganic porous nanocarriers for extremely long blood circulation in vivo. ACS Appl Mater Interfaces. 2016;8(48):32723-32731.

22. Maeda $\mathrm{H}, \mathrm{Wu} J$, Sawa $\mathrm{T}$, et al. Tumor vascular permeability and the EPR effect in macromolecular therapeutics: a review. $J$ Control Release. 2000;65(2):271-284.

23. Bu P, Chen KY, Xiang K, et al. Aldolase B-Mediated Fructose Metabolism Drives Metabolic Reprogramming of Colon Cancer Liver Metastasis. Cell Metab. 2018;27:1-14.

24. Yang N, Ye Z, Li F, et al. HPMA polymer-based site-specific delivery of oligonucleotides to hepatic stellate cells. Bioconjug Chem. 2009;20(2):213-221.

25. Adrian JE, Poelstra K, Scherphof GL, et al. Effects of a new bioactive lipid-based drug carrier on cultured hepatic stellate cells and liver fibrosis in bile duct-ligated rats. J Pharmacol Exp Ther. 2007;321(2):536-543.

26. Sato Y, Murase K, Kato J, et al. Resolution of liver cirrhosis using vitamin A-coupled liposomes to deliver siRNA against a collagen-specific chaperone. Nat Biotechnol. 2008;26(4):431-442.

27. Du SL, Pan H, Lu WY, et al. Cyclic Arg-Gly-Asp peptide-labeled liposomes for targeting drug therapy of hepatic fibrosis in rats. $J$ Pharmacol Exp Ther. 2007;322(2):560-568.

28. Suriano F, Pratt R, Tan JP, et al. Synthesis of a family of amphiphilic glycopolymers via controlled ring-opening polymerization of functionalized cyclic carbonates and their application in drug delivery. Biomaterials. 2010;31(9):2637-2645. 
29. Terada T, Iwai M, Kawakami S, et al. Novel PEG-matrix metalloproteinase-2 cleavable peptide-lipid containing galactosylated liposomes for hepatocellular carcinoma-selective targeting. J Control Release. 2006;111(3):333-342.

30. Cao Y, Gu Y, Ma H, et al. self-assembled nano particle drug delivery systems from galactosylated polysaccharide-doxorubicin conjugate loaded doxorubicin. Int J Biol Macromol. 2010;46(2):245-249.

31. Diez S, Navarro G, De ICT. In vivo targeted gene delivery by cationic nano particles for treatment of hepato cellular carcinoma. J Gene Med. 2009;11(1):38-45.

32. Qi XR, Yan WW, Shi. Hepatocytes targeting of cationic liposomes modified with soybean sterylglucoside and polyethylene glycol. $J$ Gastroenterol. 2005;11(32):4947-4952.

33. Yang KW, Li XR, Yang ZL, et al. Novel polyion complex micelles for liver-targeted delivery of diammonium glycyrrhizinate: In vitro and in vivo characterization. J Biomed Mater Res. 2009;88(1):140-148.

34. Wang Q, Zhang L, Hu W, et al. Norcantharidin-associated galactosylated chitosan nano particles for hepatocyte-targeted delivery. Nanomedicine. 2010;6(2):371-381.

35. Cho CS, Kobayashi A, Takei R, et al. Receptor-mediated cell modulator delivery to hepatocyte using nano particles coated with carbohydratecarrying polymers. Biomaterials. 2001;22(1):45-55.

36. Xie R, Xu J, Wen G, et al. The P2Y2 nucleotide receptor mediates the proliferation and migration of human hepatocellular carcinoma cells induced by ATP. J Biol Chem. 2014;289(27):19137-19149.

37. Westhaus S, Deest M, Nguyen ATX, et al. Scavenger receptor class B member 1 (SCARB1) variants modulate hepatitis $\mathrm{C}$ virus replication cycle and viral load. J Hepatol. 2017;67(2):237-245.

38. Cheon SJ, Lee CM, Kim SL, et al. Super paramagnetic iron oxide nano particles-loaded chitosan-linoleic acid nano particles as an effective hepatocyte-targe ted gene delivery system. Int $J$ Pharm. 2009;372(2):169-176.

39. Longmuir KJ, Haynes SM, Baratta JL, et al. Liposomal delivery of doxorubicin to hepatocytes in vivo by targeting heparan sulfate. Int $J$ Pharm. 2009;382(2):222-233.

40. Masakiyo H, Structure and Catalytic Properties of Carboxylesterase Isozymes Involved in Metabolic Activation of Prodrugs. Molecules. 2008;13(2):412-431.

41. Briz O, Serrano MA, Rebollo N, et al. Carriers Involved in Targeting the Cytostatic Bile Acid-Cisplatin Derivatives cis-Diamminechloro-cholyl glycinate-platinum (II) and cis-Diammine-bisursodeoxycholateplatinum (II) toward Liver Cells. Mol Pharmacol. 2002;61:853-860.

42. Wáng YJ, Baere TD, Idée JM, et al. Transcatheter embolization therapy in liver cancer: an update of clinical evidences. Chin J Cancer Res. 2015;27(2):96-112.

43. Fiume L, Busi C, Stefano G, et al. Liver targeting of adenine arabinoside mono phosphate (ara-AMP) by coupling to lactosaminated human serum albumin. Ital J Gastroenterol. 1995;27(4):189-192.

44. Beljaars L, Meijer DK, Poelstra K. Targeting hepatic satellite cells for cellspecific treatment of liver fibrosis. Front Biosci. 2002;7(3):214-222.

45. Meijer DKF, Jansen RW, Molema G. Drug targeting systems for antiviral agents: options and limitations. Antiviral Res. 1992;18(4):215-258.

46. Rensen PCN, Remco LA, Johan K. Recombinant lipoproteins: lipoprotein-like lipid particles for drug targeting. Adv Drug Deliv. 2001;47(3):251-276.
47. Martines LO, Jacquet S, Estevez JP. Ectopic b-chain of ATP synthase is apolipoprotein A-I receptor in hepatic HDL endocytosis. Nature. 2003;421(6918):75-79.

48. Huang W, Wang W, Wang P, et al. Glycyrrhetinic acid-modified poly (ethylene glycol)-b-poly(c-benzyl L-glutamate) micelles for liver targeting therapy. Acta Biomater. 2010;6(10):3927-3935.

49. Rowan SJ. Polymer self-assembly: micelles make a living. Nat Mater. 2009;8(2):89-91.

50. Branco MC, Schneider JP. Self-assembling materials for therapeutic delivery. Acta Biomater. 2009;5(3):817-831.

51. Wen J, Kim GJA, Leong KW. Poly (D, L lactide-co-ethyl ethylene phosphate)s as new drug carriers. J Control Release. 2005;6:720-725.

52. Savic R, Eisenberg A, Maysinger D. Block copolymer micelles as delivery vehicles of hydrophobic drugs: micelle-cell interactions. J Drug Target. 2006;14(6):343-355.

53. Unezaki S, Maruyama K, Takahashi N, et al. Enhanced delivery and antitumor activity of doxorubicin using long-circulating thermosensitive liposomes containing amphipathic poly ethylene glycol in combination with local hyperthermia. Pharm Res. 1994;11(8):1180-1185.

54. Lai C, Li C, Luo X, et al. Use of dual-ligand modification in Kupffer cell-targeted liposomes to examine the contribution of Kupffer cells to accelerated blood clearance (ABC) phenomenon. Mol Pharm. 2018;1-24

55. Allen TM, Hansen C, Rutledge J. Liposomes with prolonged circulation times: factors affecting uptake by reticuloendothelial and other tissues. Biochim Biophys Acta. 1989;981(1):27-35.

56. Dasgupta P, Bachhawat BK. Receptor-mediated uptake of asialoganglioside liposomes: sub-cellular distribution of the liposomal marker in isolated liver cell types. Biochem Int. 1985;10(3):327-336.

57. Spanjer HH, Scherphof GL. Targeting of lactosylceramide-containing liposomes to hepatocytes in vivo. Biochim Biophys Acta. 1983;734(1):40 47.

58. Shimizu K, Maitani Y, Takayama K, et al. Formulation of liposomes with a soybean-derived sterylglucoside mixture and cholesterol for liver targeting. Biol Pharm Bull. 1997;20(8):881-886.

59. Maitani Y, Kawano K, Yamada K, et al. Efficiency of liposomes surfacemodified with soyabean derived sterylglucoside as a liver targeting carrier in HepG2 cells. J Cont Real. 2001;75(3):381-389.

60. Drager LF, Tavoni TM, Silva VM, et al. Obstructive sleep apnea and effects of CPAP on triglyceride-rich lipoprotein metabolism. J Lipid Res. 2018;59:1027-1033.

61. Hara T, Tan Y, Huang L. In vivo gene delivery to the liver using reconstituted chylomicron remnants as a novel non viral vector. Proc Natl Acad Sci. 1997;94(26):14547-14552.

62. Valicherla GR, Dave KM, Syed AA, et al. Formulation optimization of Docetaxel loaded self-emulsifying drug delivery system to enhance bioavailability and anti-tumor activity. Sci Rep. 2016;6:26888-26895.

63. Rensen PC, Van Dijk, Havenaar EC, et al. Selective liver targeting of antivirals by recombinant chylomicrons a new therapeutic approach to hepatitis. B Nat Med. 1995;1(3):221-225.

64. Dierling AM, Zhengrong C. Targeting primaquine into liver using chylomicron emulsions for potential vivax malaria therapy. Int $J$ Pharm. 2005;303(2):143-152.

65. Rensen PCN, Havenaar DEC. Selective liver targeting of antivirals by recombinant chylomicrons a new therapeutic approach to hepatitis B. Nat Med. 1995;1(3):221-225. 
66. Wooddell CI, Rozema DB, Hossbach M, et al. Hepatocyte-targeted RNA therapeutics for the treatment of chronic hepatitis B virus infection. Mol Ther. 2013;21(5):973-985.

67. Qiao JB, Qian-QF, Xinga L. et al. Vitamin A-decorated biocompatible micelles for chemogene therapy of liver fibrosis. $J$ Control Release. 2018;283:113-125.

68. Jia Z, Gong Y, Pi Y. et al. pPB peptide-mediated siRNA-loaded stable nucleic acid lipid nano particles on targeting therapy of hepatic fibrosis. Mol Pharm. 2018;15(1):53-62.
69. Tang X, Chen L, Li A, et al. Anti-GPC3 antibody-modified sorafenibloaded nano particles significantly inhibited HepG2 hepato cellular carcinoma. Drug Delivery. 2018;25(1):1484-1494.

70. Wu JY, Wang ZX, Zhang G, et al. Targeted co-delivery of Beclin 1 siRNA and FTY720 to hepatocellular carcinoma by calcium phosphate nano particles for enhanced anticancer efficacy. International journal of nanomedicine. 2018;13:12651280. 